\title{
Heilungsvorgänge bei konservativ behandelter zystischer Knochenmarkfibrose.
}

\author{
Von Prof. Dr. Fritz Lotsch in Berlin. ${ }^{1}$ )
}

Von den krankhaften Veränderungen, die sich im Knocheninnern abspielen, müssen wir für die nachfolgenden Erörterungen folgende 3 Formen herausgreifen. Es sind dies einmal die sogenannten Knochenmarksfibrome, die sogenannten Riesenzellensarkome und die sogenannten Knochenzysten. Seit den Untersuchungen v. Recklinghausens wissen wir, daß diese anscheinend völlig heterogenen Bildungen ihre Entstehung der gleichen krankhaften Veränderung verdanken, die er als metaplastische Osteomalazie oder mit dem eingebürgerten Namen der Ostitis fibrosa bezeichnet hat.

Nach unseren heutigen Kenntnissen können wir zusammenfassend über diese Erkrankung Folgendes aussagen. Aus unbekannter Ursache kommt es namentlich in den proximalen Metaphysen der langen Röhrenknochen zu einer Proliferation der bindegewebigen Komponente des Knochenmarks, die sich auf Kosten einmal der hämatogenen Komponente, zum andern des Knochengewebes Raum schafft. Da der entzündliche Charakter der Erkrankung keineswegs völlig sicher ist, ziehe ich die Bezeichnung, ,proliferierende Markfibrose" vor. Beteiligt sich nur das fibröse Knochenmarksstroma an der herdförmigen Wucherung, so kommt es zur Bildung der seltenen reinen Knochenmarksfibrome. Haufig nehmen jedoch die Knochenmarksriesenzellen, die wir auch unter dem Namen der Myeloplaxen und besonders der Osteoklasten kennen, an der Wucherung teil, sodaß sie vielfach das histologische Bild beherrschen. So haben wir das sogenannte Riesenzellensarkom vor uns, dessen häufigste Erscheinungsform die bekannte Epulis ist. Reichlicher Gehalt an Blutpigment gibt diesen Tumoren eine rost- bis schwarzbraune Färbung, die ihnen den sehr bezeichnenden Namen der braunen Knochenmarkstumoren besonders im Lager der Chirurgen eingetragen hat. Die beiden genannten Tumorarten neigen zur Kolliquation. Das gilt besonders für die reinen Fibrome und mag der Grund dafür sein, daB diese Geschwulstform so sehr selten zur Beobachtung kommt. Die kleinen zystischen Hohlräume konfluieren allmählich zu größeren, was durch den Befund von Septen und deren Resten auch bei den größeren Knochenzysten noch det.tlich erkennbar bleibt. Die makro-

1) Nach einem Vortrag an 9. 11. 1920 in der Berliner Gesellschaft für Chirurgie. 
skopisch auBerordentlich glatt, wie lackiert aussehende Wand der Zysten wird durch die Reste des fibrösen Gewebes gebildet, eine Epithel- oder Endothelauskleidung fehlt mit völliger Sicherheit, sodaB es sich eigentlich um sogenannte falsche Zysten handelt. Der Zysteninhalt ist seröse Flüssigkeit, die je nach dem Grad der Blutbeimengung bald gelblich, bald rostbraun, bald rein blutig erscheint. Der Zysteninhalt entleert sich bei der. Eröffnung gewöhnlich unter Druck, d. h. im Innern der Zyste sclieint ein Gewebsüberdruck zu bestehen.

Die Wachstumsenergie der durch die Markfibrose bedingten Gebilde findet eine, wie es scheint, absolute Grenze am Periost, das erfahrungsgemä $\beta$ am Krankheitsproze $B$ in keiner Weise beteiligt ist "und auch, wenn überhaupt, so doch in sehr geringem Grade zu einem verstärkten Knochenanbau gereizt wird. Da die Erkrankung meist jugendliche Individuen befällt und, wie bereits erwähnt, die Gegend der Metaphyse der Lieblingssitz der Erkrankung ist, so tritt sie auch in Beziehung zum Epiphysenknorpel. Während nun die reinen Fibrome und die aus ihnen hervorgegangenen Zysten auch den Epiphysenknorpel' als Grenze respektieren, ist anscheinend die Wachstumsenergie der Riesenzellenfibrome größer; sie durchbrechen ihn und finden erst am intakt bleibenden Gelenkknorpel ihre absolute Orenze.

Die Krankheit tritt entweder solitär in nur einem Knochen oder als generalisierte Form der eigentlichen v. Reckling ha us e nschen Knochenkrankheit auf. Uns beschäftigt im Folgenden besonders die solitäre Form.

Der erkrankte Knochen wird durch den verstärkten Knochenabbau seiner Kortikalis in zunehmendem Maße stellenweise völlig beraubt und verliert infolgedessen seine Festigkeit. Das Zustandekommen von pathologischen Frakturen nimmt deshalb nicht wunder. In der Anamnese spielt ein Trauma gewöhnlich eine große Rolle und wird als ursächliches Moment gewertet. Daß dies ein Irrtum ist, geht schon aus der einfachen Ueberlegung hervor, daß die zahllosen Knochenbrüche nie von einer proliferierenden Markfibrose gefolgt sind, ferner daraus, daB das Röntgenbild in allen jenen Fällen von Markfibrose mit pathologischer Fraktur zur Zeit der Fraktur bereits eine ausgebreitete Rarefikation des Knochens im Röntgenbilde mühelos nachweisen läßt. . Der Röntgenbefund ist bei der Markfibrose, besonders bei größeren Knochenzysten, sehr typisch. Abgesehen von dem mehr oder -minder vorgeschrittenen Schwund der Kortikalis, interessiert besonders das Verhalten des Periostes, das keineriei osteophytäre Bildungen aufweist und sich auch im Röntgenbifde als völlig unbeteiligt am Krankheitsprozeß darstellt. Erfahrungsgemäß heilen auch trotz bestehender Markfibrose die pathologischen Frakturen anstandslos.

Es ist eine seit langem bekannte Tatsache, da $B$ die Prognose der solitären Markfibrose sehr günstig ist und daB davon auch die nach ihrem histologischen Bilde als Riesenzellensarkome bezeichneten Tumoren bis auf gelegentliche Lokalrezidive keine Ausnahme machen. Die Freilegung des Herdes und die gründliche Exkochleation genügt erfahrungsqgemäB zur Beseitigung des Leidens. Man kann sich beim Verfol'g des Heilungsverlaufs des Eindrucks nicht erwehren, daB das unbeteiligte Periost durch die Eröffnung der wohl tatsächlich unter einem gewissen Gewebsüberdruck stehenden Markfibrose geradezu entfesselt wird und nunmehr in kürzester Zeit die Regeneration des zerstörten Knochengewebes zu übernehmen in der Lage ist. Trotz der längst bekannten guten Prognose der geschwulstartigen und zystischen Bildungen der Markfibrose erscheinfes nach manchen Veröffentlichungen auch der letzten Zeit nicht überflüssig, auf die genügende therapeutische Wirksam. keit der Exkochleation nochmals mit Nachdruck hinzuweisen. Die Resektion des erkrankten Knochens ist wach unseren heutigen Kenntnissen auch in vorgeschrittenen Fällen nicht $z u$ rechtfertigen. Die Amputation gilt längst als obsolet. Zur Veranschaulichung der Heilungsvorgänge bei einer solitären Knochenzyste des oberen Humerusdrittels gestatte ich mir Ihnen eine fortlaufende Serie von Röntgenbildern zu projizieren, die von einem im Dezember 1918 von mir operierten 10 jährigen Mädchen stammt. Der Fall ist auf meine Veranlassung in elner Inauguraldissertation von Arthur $\mathrm{Ha}$ aring im April 1919 unter dem Titel "Ueber Knochenzysten" in extenso veröffentlieht worden. Gelegentlich einer im Jahre vorher eingetretenen Fraktur des rechten Oberarms wurde durch das Röntgenbild bereits die krankhafte Markfibrose festgestellt. Die in 10 tägigen Abständen aufgenommenen Röntgenbilder ergeben einen zunehmenden Kalkgehalt der erkrankten Knochenpartie. Die nach 10 Wochen noch bestehende längliche Aufhellung im Schaftinnern der oberen Humerushälfte erweist sich 9 Monate nach der Verletzung als völlig mit kalkhaltiger Knochensubstanz ausgefüllt und zeigt durch ihre intensive Schwärzung im Diapositiv einen wesentlich stärkeren Kalkgehalt als die normal röhrenhaltige untere Schafthälfte des Humerus. Die Markraumbildung in dem erkrankten, seinerzeit bis zum Epiphysenknorpel reichenden Knochenabschnitt ist auch auf dem letzten, 11 Monate nach der Operation aufgenommenen Bilde noch nicht wahrzunehmen. Dagegen ist die Abknickung der früheren pathologischen Fraktur in idealer Weise ausgeglichen. Ob es im weiteren Verlauf zu einer normalen Restitution der Markhöhle kommen wird, ist interessant, und diese Feststellung soll nicht verabsäumt werden. Da bei der Operation der Epyphysenknorpel geschont werden konnte, ist eine Wachstumsstörung nicht eingetreten, die Funktion des Armes völlig unbehindert 